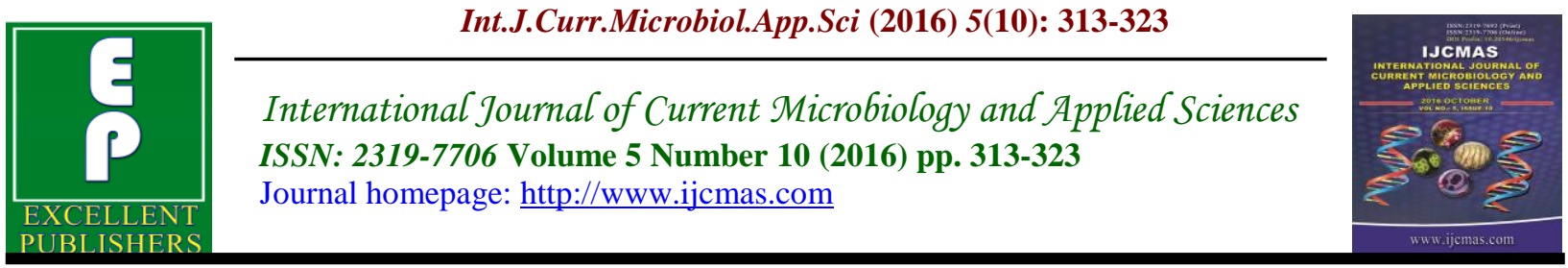

Original Research Article

http://dx.doi.org/10.20546/ijcmas.2016.510.035

\title{
Incidence of VAP in Tertiary Care Hospital
}

\author{
Ranjeeta Kumari ${ }^{1}$, Prakash C. Choudhary ${ }^{2}$ and Bhanwar Lal Jat ${ }^{3 *}$ \\ ${ }^{1}$ Department of Biotechnology, Mewar University, Gangrar, Chittorgarh, Rajasthan, India \\ ${ }^{2}$ Department of Chemistry, Mewar University, Gangrar, Chittorgarh, Rajasthan, India \\ ${ }^{3}$ Department of Agriculture Biotechnology, Bhagwant University Ajmer, Rajasthan, India \\ *Corresponding author
}

Keywords

VAP, nosocomial pneumonia,

Hospital-acquired pneumonia

(HAP).

\section{Article Info}

Accepted:

14 September 2016

Available Online:

10 October 2016

\section{A B S T R A C T}

Pneumonia is the second most common nosocomial infection in the United States and is a leading cause of death due to hospital-acquired infections. Ventilatorassociated pneumonia (VAP) is a form of nosocomial pneumonia that occurs in patients receiving mechanical ventilation for longer than 48 hours. The incidence of VAP is $22.8 \%$ in patients receiving mechanical ventilation, and patients receiving ventilator support account for $86 \%$ of the cases of nosocomial pneumonia. Furthermore, the risk for pneumonia increases 3- to 10-fold in patients receiving mechanical ventilation. Ventilator-associated pneumonia (VAP) is a major cause of hospital morbidity and mortality despite recent advances in diagnosis and accuracy of management. However, as taught in medical science, prevention is better than cure is probably more appropriate as concerned to VAP because of the fact that it is a well preventable disease and a proper approach decreases the hospital stay, cost, morbidity and mortality. The aim of the study is to critically review the incidence and outcome, identify various risk factors and conclude specific measures that should be undertaken to prevent VAP. We studied patients randomly, kept on ventilator support for more than $48 \mathrm{~h}$.

\section{Introduction}

Ventilator-associated pneumonia (VAP) refers to bacterial pneumonia developed in patients who have been mechanically ventilated for duration of more than $48 \mathrm{~h}$. It ranges from 6 to $52 \%$ and can reach $76 \%$ in some specific settings. Hospital-acquired pneumonia (HAP) is the pneumonia after 48 $\mathrm{h}$ or more after admission, which did not appear to be incubating at the time of admission. The presence of HAP increases hospital stay by an average of 7-9 days per patient also imposes an extra financial burden to the hospital. The risk of VAP is highest early in the course of hospital stay, and is estimated to be 3\%/day during the first 5 days of ventilation, 2\%/day during days $5-10$ of ventilation and $1 \%$ /day after this. Interventions to prevent VAP begin at the time of intubation and should be continued until extubation. With the extreme shortage of nurses and the resultant increase in the number of less experienced nurses in the intensive care unit, education on the prevention of VAP is essential, because the 
occurrence of nosocomial infections is directly related to the adequacy of staff. Nurses need to understand the pathophysiology of VAP, risk factors for this type of pneumonia, and strategies that may prevent the disease. The onset of VAP can be divided into 2 types: early and late (Cook et al., 1998). Early-onset VAP occurs 48 to 96 hours after intubation and is associated with antibiotic-susceptible organisms (Niederman, 1990). Late-onset
VAP occurs more than 96 hours after intubation and is associated with antibioticresistant organisms. Interventions to prevent VAP should begin at the time of, or if possible, before intubation. The pathophysiology of VAP involves 2 main processes: colonization of the respiratory and digestive tracts and micro aspiration of secretions of the upper and lower parts of the airway.

\section{Risk factors for ventilator associated pneumonia}

\begin{tabular}{|l|l|}
\hline Host Factors & Intervention factors \\
\hline Age $>60$ & Duration of MV \\
\hline Severity of illness & Reintubation \\
\hline Organ failure & PEEP \\
\hline Nutritional state or hypoalbuminemia & Frequent ventilator circuit changes \\
\hline Upper abdominal or thoracic surgery & Nasogastric tube \\
\hline Chronic lung disease & Intracranial pressure monitoring \\
\hline Neuromuscular disease & Paralytic agents or sedation \\
\hline Coma and depressed level of consciousness & H2 blockers +- antiacids \\
\hline Large volume aspiration & Supine head position \\
\hline Upper respiratory tract colonization & Transport out of the ICU \\
\hline Sinusiitis & \\
\hline
\end{tabular}

Bacteria associated with ventilatorassociated pneumonia

Colonization of bacteria refers to the presence of bacteria without an active host response. Bacterial colonization of the lungs can be due to spread of organisms from many different sources, including the oropharynx, sinus cavities, nares, dental plaque, gastrointestinal tract, patient-topatient contact, and the ventilator circuit. Inhalation of colonized bacteria from any of these sources can cause an active host response and, ultimately, VAP. The presence of an endotracheal tube provides a direct route for colonized bacteria to enter the lower respiratory tract. Upper airway and oral secretions can pool above the cuff of an endotracheal tube and line the tube, forming a biofilm. Starting as early as 12 hours after intubation, the biofilm contains large amounts of bacteria that can be disseminated into the lungs by ventilatorinduced breaths. In addition, the biofilm may become dislodged by instillation of saline into the endotracheal tube, suctioning, coughing, or repositioning of the endotracheal tube. Endotracheal tubes cause an abnormal interruption between the upper airway and the trachea, bypassing the structures in the upper airway and providing bacteria a direct route into the lower airway. Because the upper airway is bypassed, a decrease occurs in the body's ability to filter and humidify air. In addition, the cough reflex is often eliminated and/or decreased by the presence of an endotracheal tube, and mucociliary clearance can be impaired because of mucosal injury during intubation. An endotracheal tube provides a place for bacteria to bind in the trachea, a situation that further increases production and 
secretion of mucus. The impairment of these natural host defense mechanisms increases the likelihood of bacterial colonization and subsequent aspiration of the colonized organisms. Aspiration of gastric contents is another potential cause of VAP, because the stomach serves as a reservoir for bacteria. Most patients receiving mechanical ventilation have a nasogastric or an orogastric tube in place for enteral feedings and administration of medications or for gastric decompression. The presence of a nasogastric or an orogastric tube interrupts the gastroesophageal sphincter, leading to increased gastrointestinal reflux and providing a route for bacteria to translocate to the oropharynx and colonize the upper airway. Enteral feedings increase both gastric $\mathrm{pH}$ and gastric volume, increasing the risk of both bacterial colonization and aspiration.

\section{Etiologic agents}

The causative organism vary according to the patients demographics in the ICU, methods of diagnosis, the durations of hospital and ICU stays, and the antibiotics policy. In NNIS data, although the most frequent reported isolate was Staphylococcus aureus (17\%) and 59\% of all reported isolates were gram negative bacteria.

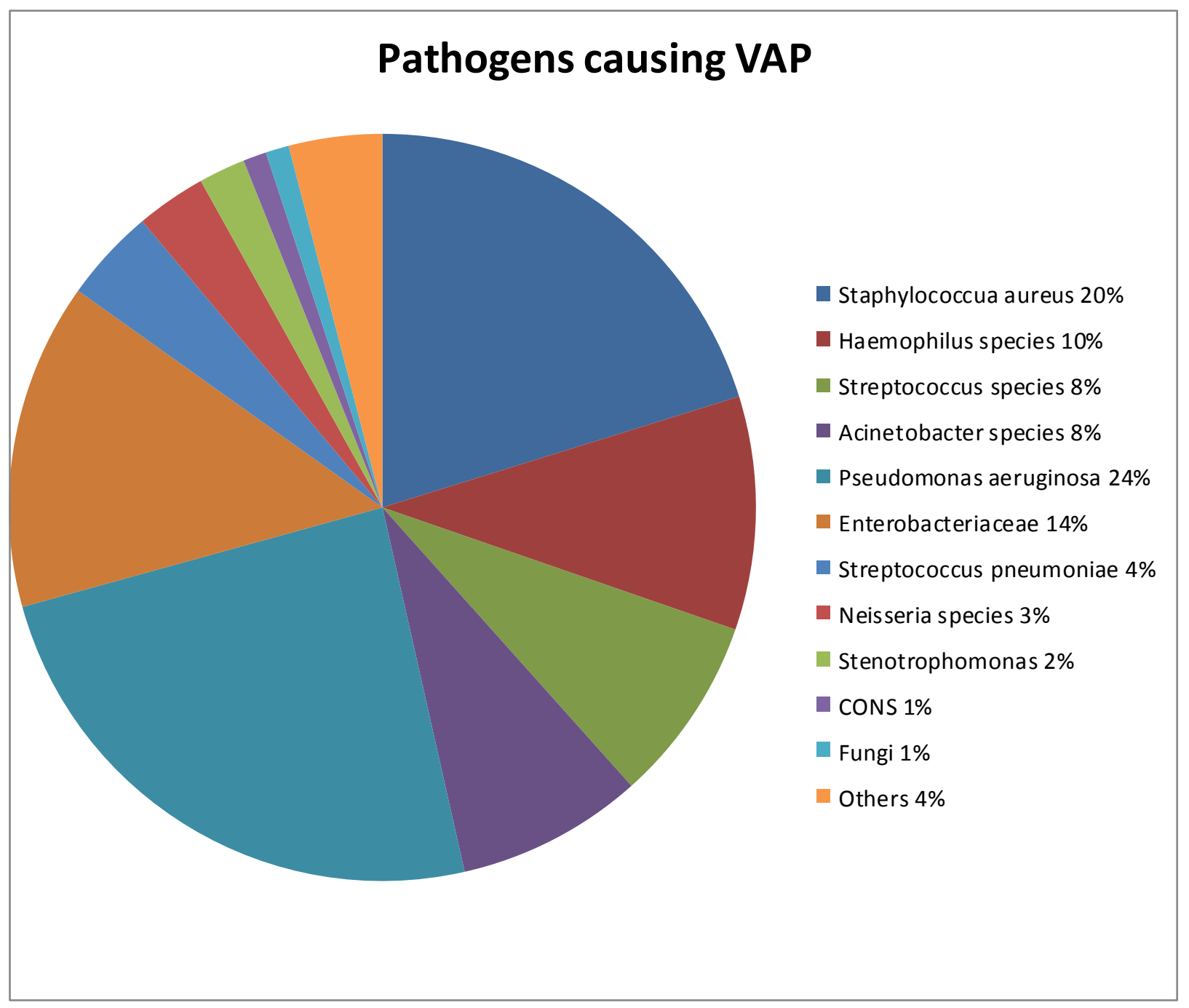


The most common pathogens are the ESKAPE bugs- (ESKAPE bugs refers to) (i) Enterococcus faecium, (ii) Staphylococcus aureus, (iii) Klebsiella pneumoniae, (iv) Acinetobacter baumanii,

Pseudomonas aeruginosa and

\section{Pathogens expected in diseased condition}

\begin{tabular}{|l|l|}
\hline Streptococcus pneumonia & Smoking, COPD, absence of antibiotic therapy \\
\hline MSSA & Younger age, traumatic coma, Neurosurgery \\
\hline MRSA & $\begin{array}{l}\text { COPD, steroid therapy, longer duration of MV, } \\
\text { prior antibiotics }\end{array}$ \\
\hline Acinetobacter species & ARDS, Head trauma, neurosurgery \\
\hline Pseudomonas aeruginosa & $\begin{array}{l}\text { COPD, steroid therapy, longer duration of MV, } \\
\text { prior antibiotics }\end{array}$ \\
\hline
\end{tabular}

\section{Clinical Diagnosis}

Clinical diagnosis has been criticized to have poor accuracy and reliability. Thus, the Centers for Disease Control and Prevention has introduced a new definition based upon objective and recordable data. Institutions are nowadays reporting a VAP zero rate in surveillance programs, which is in discrepancy with clinical data. This reduction has been highlighted in epidemiological studies, but it can only be attributed to a difference in patient selection, since no additional intervention has been taken to modify pathogenic mechanisms in these studies. The principal determinant of VAP development is the presence of the endotracheal tube (ETT). Contaminated oropharyngeal secretions pool over the ETT cuff and subsequently leak down to the lungs through a hydrostatic gradient

\section{Microbiologic Diagnosis}

\section{Blood and pleural fluid cultures}

Although VAP spreads to the blood or pleural space in $<10 \%$ of cases, if an organism known to cause pneumonia is cultured in the setting of clinically suspected pneumonia, treatment is warranted.
Consequently, most experts recommend that two sets of blood cultures and a thoracentesis for nonloculated pleural effusions of $\geq 10 \mathrm{~mm}$ in diameter on a lateral decubitus chest radiograph should be part of the evaluation of suspected VAP. If the effusion is loculated, ultrasound guidance may be required. However, it is important to keep in mind not only that the sensitivity of blood cultures for the diagnosis of VAP is less than $25 \%$ but also that when positive, the organisms may originate from an extrapulmonary site of infection in as many as $64 \%$ of cases and even when VAP is present

\section{Nonquantitative or semiquantitative airway sampling}

Gram staining and nonquantitative and semiquantitative cultures of tracheal secretions have the advantages of reproducibility and of requiring little technical expertise and no specialized equipment or technique. However, these studies add little to the sensitivity and specificity of the clinical diagnosis of VAP, as the upper respiratory tract is rapidly, within hours of intubation, colonized by potential pulmonary pathogens, even when pneumonia is not present. Thus, if an 
organism is cultured or noted on Gram stain, one does not know if it is the cause of the pneumonia or simply colonization. In a study of 48 patients with respiratory failure, concordance between tracheal nonquantitative cultures and cultures of lung tissue from open lung biopsy was only $40 \%$. In that study, of those patients with pneumonia on lung histology, endotracheal aspirate (ETA) had a sensitivity of $82 \%$ but a specificity of only $27 \%$. In addition, routine surveillance cultures of ETAs to anticipate the etiology of a subsequent pneumonia can be misleading in a significant percentage of patients, though recent data indicate that quantitative ETAs may be here

\section{Quantitative cultures of airway specimens}

To potentially improve the specificity of the diagnosis of VAP and the consequent unnecessary antibiotic use and its associated problems, numerous studies have investigated the role of quantitative cultures of respiratory secretions.

These have included nonbronchoscopic methods such as quantitative cultures of ETAs (QEAs) and sampling of secretions from distal airways "blindly" via an endobronchial catheter. Blind bronchial sampling (BBS), PSB, protected telescoping catheter (PTC), BAL, and protected BAL (mini-BAL) samples can be obtained via the latter method. Bronchoscopic sampling methods permit quantitative cultures of PSB, PTC, and protected and nonprotected BAL specimens.

\section{Materials and Methods}

This study was conducted in medical ICU of tertiary care hospital for 1 year. The study was approved by the hospital infection control members. We enrolled patients from
24 to 80 yrs, who required ventilator support for the preceding $48 \mathrm{hrs}$ for each patient prevention ventilator bundle checklist by CDC was followed, which include 7 strategies to reduce VAP incidence and mortality rate.

Also for each patient below parameters recorded:-

- Age

- Gender

- Primary diagnosis on admission

- Reason of ventilation

- Comorbid conditions(COPD, cardiac respiratory disease disease, alcholoism etc)

- Duration of mechanical ventilation

- Antibiotic received before sampling

- Radiographic (X- Ray report)

In each patient only invasive sample (ETT Lavage) were collected by junior residents or by staff nurses.

\section{Sample collection}

\section{Procedure}

The ETT sample lavage sample were collected via a sputum suction trap. A sterile suction catheter inserted. Then normal saline was instilled into distal airways. Aspirate was collected in sterile container. Samples were transported for bacteriological examination and cultures in microbiology laboratory. Further processing was done in laboratory by us.

\section{Sample processing}

\section{Procedure (for gram negative organism)}

10 microliter (loopful) of sample transferred on to dehydrated media plates on mc conkey agar plate. Make smear of sample for gram 
staining. Streaked with sterile inoculation loop. Plates then inoculated at $37^{0}$ centigrade for $24 \mathrm{hrs}$. After $24 \mathrm{hrs}$ of inoculation see the growth on mc conkey agar plates, if significant growth found then passed colonies into peptone water[Perform oxidase test of colonies if colonies are NLF (non lactse fermenting)]. Incubate the tubes of peptone water at 37 centigrade for $4 \mathrm{hrs}$. Apply biochemicals and incubate for $24 \mathrm{hrs}$ at $37^{\circ}$ centigrade. Next day add reagents of biochemicals like kovac's reagent (indole), biurret reagent (voges proskaeur)

\section{Procedure (for gram positive organism)}

10 microliter (loopful) of sample transferred on to dehydrated media plates on blood agar. Make smear of sample for gram staining. Streaked with sterile inoculation loop. Plates then incubated at $37^{\circ}$ centigrade for $24 \mathrm{hrs}$. After 24 hrs of incubation see the growth on blood agar plates, if significant growth found then passed colonies into glucose broth (perform catalase and coagulase test when white opaque colonies present). Incubate the tubes of glucose broth at 37 centrigrade for $4 \mathrm{hrs}$. Apply sensitivity on muller hinton agar.

\section{Antibiotic Management}

The ATS has recently published guidelines to guide empirical antibiotic choices. These guidelines are divided into those for patients at risk for VAP caused by multidrugresistant organisms and those for patients without such risk. Risk factors for multidrug-resistant organisms include prior antimicrobial therapy in the preceding 90 days, current hospitalization exceeding 5 days (not necessarily ICU days), high frequency of resistance in the community or local hospital unit, and immunosuppressive disease and/or therapy. In addition, the clinician must consider risk factors for health care-associated pneumonia, as such a pneumonia may present with multidrugresistant organisms even upon hospital admission. Such risk factors for the intubated patient include a hospitalization for $>2$ days within the preceding 90 days, residence in a long-term care facility, chronic dialysis within 30 days, home wound care, home infusion therapy (inclusive of antibiotics), and a family member with a multidrug-resistant pathogen. In the absence of risk factors for multidrug-resistant bacteria, the clinician should choose empirical therapy for Streptococcus pneumoniae, Haemophilus influenzae, methicillin-sensitive Staphylococcus aureus, and antibioticsensitive gram-negative enteric organisms. Antibiotic choices include ceftriaxone, quinolones (levofloxacin, moxifloxacin, or ciprofloxacin), ampicillin/sulbactam, or ertapenem. When risk factors for multidrugresistant organisms are present, the clinician must consider not only the organisms listed above but also Pseudomonas aeruginosa, Klebsiella, Enterobacter, Serratia, Acinetobacter, Stenotrophomonas maltophilia, Burkholderia cepacia, and methicillin-resistant S. aureus. Empirical therapy is broadened to include (i) either an antipseudomonal cephalosporin (cefepime or ceftazadime), an antipseudomonal carbepenem (imipenem or meropenem), or a $\beta$-lactam $/ \beta$-lactamase inhibitor (pipercacillin-tazobactam) plus (ii) an antipseudomonal fluoroquinolone (ciprofloxacin or levofloxacin) or an aminoglycoside (amikacin, gentamicin, or tobramycin) plus linezolid or vancomycin.

\section{Results and Discussion}

This study is done for 1 year in tertiary care hospital. The results are given table.1,2 below:- 
Table.1

\begin{tabular}{|l|l|}
\hline Points & Count \\
\hline Total number of patients & 48 \\
\hline Sample received & 48 \\
\hline Positive cultures & 34 \\
\hline Sterile & 14 \\
\hline Positive case for VAP & 17 \\
\hline Expired in total & 31 \\
\hline
\end{tabular}

Table.2 Organism isolated

\begin{tabular}{|l|l|}
\hline Organism & Count \\
\hline Klebesiella & 11 \\
\hline E.coli & 2 \\
\hline Acinetobacter & 5 \\
\hline Pseudomonas & 3 \\
\hline Total & 17 \\
\hline
\end{tabular}

\section{No. of patients diagnosed VAP}

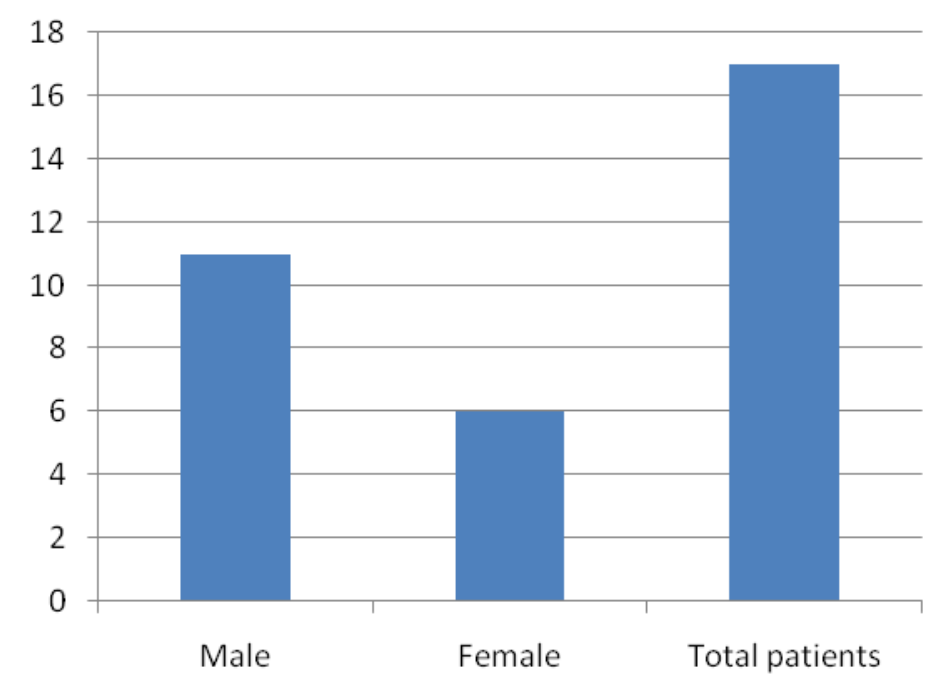

No. of patients diagnosed VAP 


\section{No. of patients in months}

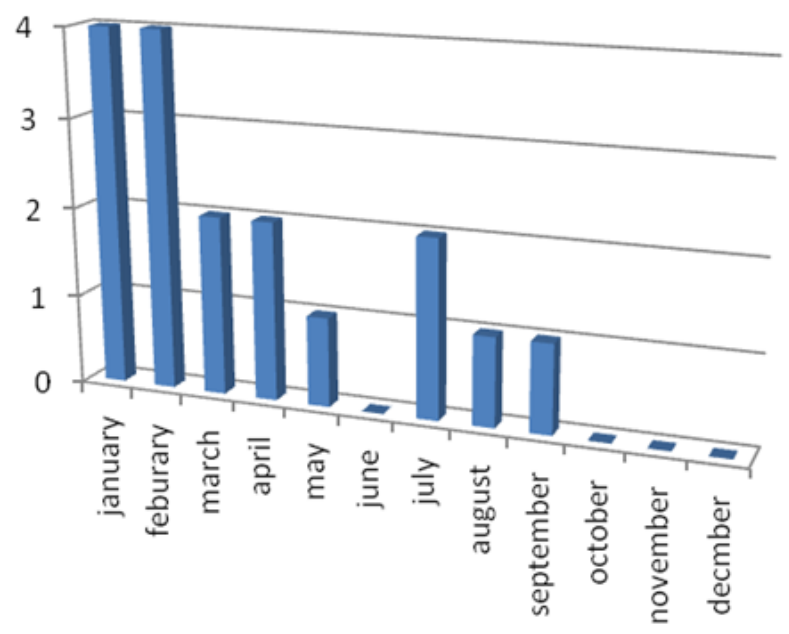

No. of patients in months

\section{Site of sample}

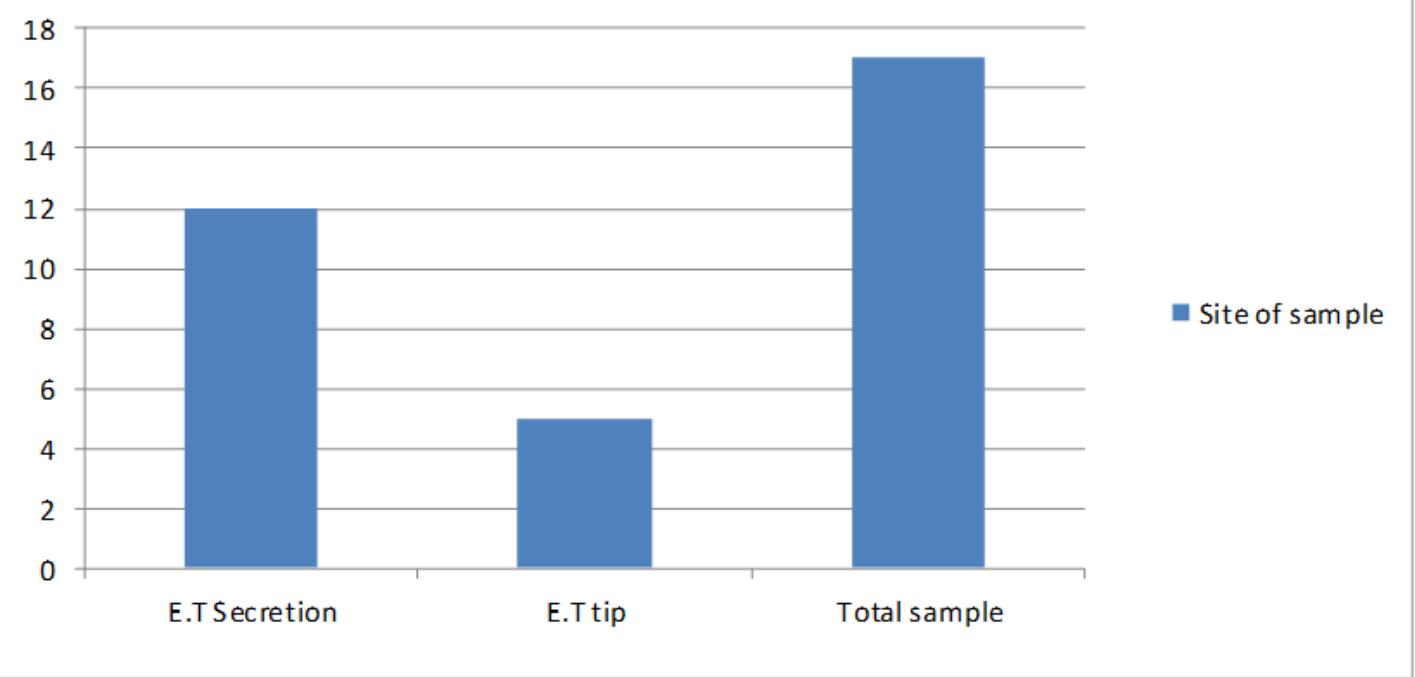



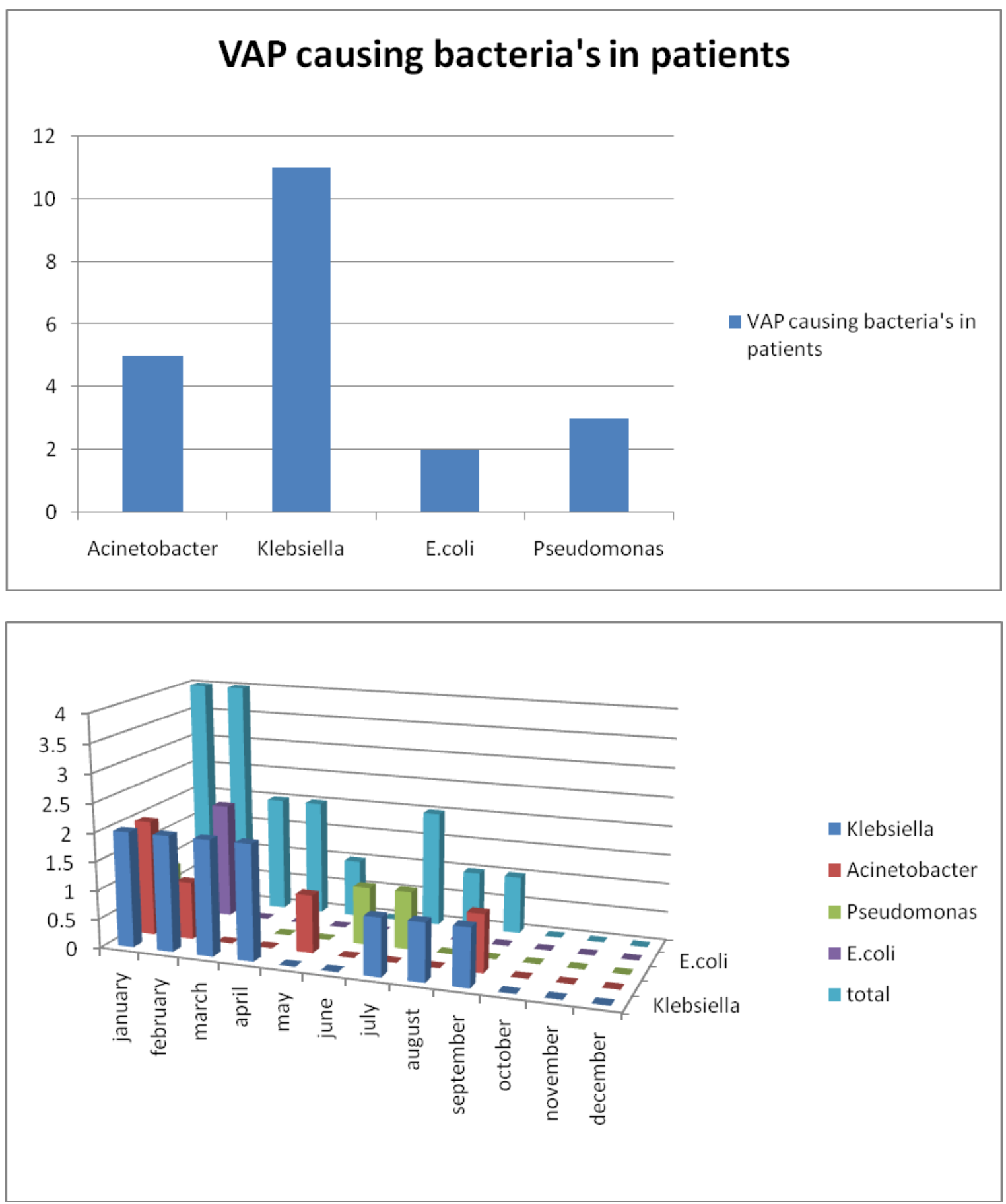

Ventilator days $=100$

Patient on ventilator $=48$

Positive VAP cases $=17$

$\mathrm{VAP}$ rate $=$

No. of VAP cases/ ventilator days*100

$17 / 100 * 100=17 \%$ 
In the study of our set up, males predominated (62\%). Although the incidence of VAP was also high in males, it was statistically not significant. The incidence of VAP in our setting was 37\%. In the era of advanced diagnosis and early management of possible complications, the incidence tends to be lower. In recent studies, the reported incidence is very low, ranging from 15 to $30 \%$. The high incidence in our study may be due to a lower number of cases (i.e., 100) and lack of adequate nursing staff (which should ideally be 1:1 as compared to 4:1 in our institute) which may have adversely affected the quality of care given to the patients. The most common organism associated with VAP is Klebsiella (43.24\%), followed by Pseudomonas (18.91\%). Also, the overall mortality rate was high in the Pseudomonas group $(62.5 \%)$. In other studies, isolation of Pseudomonas ranges from 15 to $25 \%$. Susceptibility testing could not be studied in all patients due to a lack of clinical microbiologic support as it is not done routinely and sending samples outside is not allowed by the hospital authority except in special cases. Hand washing is widely recognized as an important but underused measure to prevent nosocomial infections. According to the 2004 CDC (Center for Disease Control) guidelines, hands should be washed before and after patient contact and also in between patient contact. Chlorhexidine has been shown to be effective in the control of ventilator-circuit colonization and pneumonia caused by antibiotic-resistant bacteria. Oropharyngeal decontamination with Chlorhexidine solution has also been shown to reduce the occurrence of VAP in patients undergoing cardiac surgery. VAP, although often preventable, has a large impact on morbidity and mortality. Together with other healthcare providers, nurses play a key role in preventing VAP. Many of the interventions are part of routine nursing care. Education for all healthcare providers should focus on the risk factors for VAP and on preventive measures. In order to further decrease the incidence of VAP, protocols and monitoring tools must be developed. VAP is not a new diagnosis, but education and research on the prevention of this life-threatening problem are ongoing.

\section{References}

Apostolopoulou, E., Bakakos, P., Katostaras, T., Gregorakos, L. 2003. Incidence and risk factors for ventilator-associated pneumonia in 4 multidisciplinary intensive care units in Athens, Greece. Respir. Care, 48: 681-8.

Cook, D.J., Walter, S.D., Cook, R.J., Griffith, L.E., Guyatt, G.H., Leasa, D., et al. 1998. Incidence of and risk factors for ventilator-associated pneumonia in critically ill patients. Ann. Intern Med., 129: 440.

Diaz, E., Rodriguez, A.H,. Rello, J. 2005. Ventilator-associated pneumonia: issues related to the artificial airway. Respir. Care, 50: 900-6.

Doebbeling, B.N., Stanley, G.L., Sheetz, C.T., Pfaller, M.A., Houston, A.K., Annis, L., et al. 1992. Comparative efficacy of alternative hand-washing agents in reducing nosocomial infections in intensive care units. $N$. Engl. J. Med., 327: 88-93.

Fagon, J.Y., Chastre, J., Wolff, M., Gervais, C., Parer-Aubas, S., Stephan, F., et al. 2000. Invasive and noninvasive strategies for management of suspected ventilator-associated pneumonia: a randomized trial. Ann. Intern Med., 132: 621-30.

Kollef, M.H. 1993. Ventilator-associated pneumonia: A multivariate analysis. JAMA, 270: 1965-70. 
Kollef, M.H., Von Harz, B., Prentice, D., Shapiro, S.D., Silver, P., St John, R., et al. 1997. Patient transport from intensive care increases the risk of developing ventilator-associated pneumonia. Chest, 112: 765-73.

Niederman, M.S. 1990. Gram-negative colonization of the respiratory tract: pathogenesis and clinical consequences. Semin. Respir. Infect., 5: $173-84$.

Rumbak, M.J., Cancio, M.R. 1995. Significant reduction in methicillin- resistant Staphylococcus aureus ventilator-associated pneumonia associated with the institution of a prevention protocol. Crit. Care Med., 23: $1200-3$.

Torres, A., Gatell, J.M., Aznar, E., elEbiary, M., Puig, de la Bellacasa, J., Gonzalez, J., et al. 1995 . Reintubation increases the risk of nosocomial pneumonia in patients needing mechanical ventilation. Am. $J$. Respir. Crit. Care Med., 152: 137-41.

\section{How to cite this article:}

Ranjeeta Kumari, Prakash C. Choudhary and Bhanwar Lal Jat. 2016. Incidence of VAP in Tertiary Care Hospital. Int.J.Curr.Microbiol.App.Sci. 5(10): 313-323.

doi: http://dx.doi.org/10.20546/ijcmas.2016.510.035 